\title{
Criação e Implantação de Programa de Mestrado Profissional vinculado à Residência Médica: a experiência da Faculdade de Medicina de Ribeirão Preto da Universidade de São Paulo
}

\author{
Design and Implementation of a Professional Master's Program linked to the \\ Medical Residency: the experience of the Ribeirão Preto Medical School, \\ University of São Paulo
}

Cristina Marta Del-Ben ${ }^{1,2}$ (D), Valdes Roberto Bollela ${ }^{1,3}$ (D), Ana Paula de Carvalho Panzeri Carlotti ${ }^{1,4}$ (D), Luciane Loures dos Santos ${ }^{1,5}$ (D), Edgard Eduard Engel ${ }^{1,6}$ (D), Edwaldo Edner Joviliano ${ }^{1,7}$ (D), João Marcello Furtado ${ }^{1,8}$ (D), Júlio César Rosa e Silva ${ }^{1,9}$ (it)

\begin{abstract}
RESUMO
A prática assistencial em saúde apresenta desafios crescentes, exigindo do médico o desenvolvimento de habilidades e competências diversificadas que facilitem a sua atuação qualificada. A reflexão crítica e a autonomia no gerenciamento da prática profissional exigem do médico domínio de fundamentos de metodologia científica, o que nem sempre é contemplado nas matrizes curriculares dos programas de residência médica, por seu caráter de treinamento em serviço. Visando suprir essa lacuna na formação médica, foi instituído, na Faculdade de Medicina de Ribeirão Preto da Universidade de São Paulo (FMRP-USP), um programa de pós-graduação stricto senso (mestrado profissional) vinculado a programas de pós-graduação lato sensu (residência médica e estágios de complementação especializada) do Hospital das Clínicas da FMRP-USP. Este relato de experiência destina-se à apresentação do racional e dos processos para a implantação do Programa de Pós-Graduação "Mestrado Profissional em Medicina" da FMRP-USP, bem como seus objetivos, público-alvo e perfil do egresso. Espera-se que este relato contribua para a reflexão a respeito dos diferentes componentes e da complexidade da formação médica.
\end{abstract}

Palavras-chave: Pós-graduação, Mestrado profissional, Residência médica

\begin{abstract}
The health care practice presents growing challenges, requiring physicians to develop various skills and competencies that facilitate their qualified performance. Critical reflection and autonomy in the management of professional practice require the physician to master the fundamentals of scientific methodology, which is not always included in the curriculum of medical residency programs due to its in-service training nature. Aiming to fill this gap in medical education, a graduate program (professional master's degree) was established at the Ribeirão Preto Medical School at the University of São Paulo (FMRP-USP). This experience report is intended to present the rationale and processes for implementing the Graduate Program "Professional Master in Medicine" at the FMRP-USP, in addition to its objectives, target audience, and graduate profile. It is expected that this report will contribute to a reflection on the different components and complexity of medical training.
\end{abstract}

Keywords: Graduate program, Professional master, Medical residency

\footnotetext{
Universidade de São Paulo, Faculdade de Medicina de Ribeirão Preto (FMRP-USP), Programa de Pós-Graduação Mestrado Profissional em Medicina (SP), Brasil

Departamento de Neurociências e Ciências do Comportamento, FMRP-USP

Departamento de Clínica Médica, FMRP-USP

Departamento de Puericultura e Pediatria, FMRP-USP

Departamento de Medicina Social, FMRP-USP

Departamento de Ortopedia e Anestesiologia, FMRP-USP

Departamento de Cirurgia e Anatomia, FMRP-USP

Departamento de Oftalmologia, Otorrinolaringologia e Cirurgia da Cabeça e Pescoço, FMRP-USP

Departamento de Ginecologia e Obstetrícia, FMRP-USP
} 


\section{CONTEXTUALIZAÇÃO DO PROBLEMA}

A complexidade e alta demanda da assistência em saúde apontam para uma necessidade crescente, nos setores públicos e privados, de profissionais efetivamente habilitados para o desenvolvimento e a incorporação de novos conhecimentos científicos à prática profissional. As demandas da sociedade atual exigem um profissional de saúde capaz de interferir nas práticas assistenciais e modificar seu ambiente de trabaIho, integrando o conhecimento científico e novas tecnologias em saúde com as necessidades e peculiaridades locais. É desejável, portanto, que profissionais de saúde possuam capacidade real de análise crítica e reflexiva da produção científica e tecnológica na área médica. Ademais, um profissional com uma qualificação diversificada também contribui para a otimização da aplicação de recursos humanos e financeiros.

Tradicionalmente, a formação médica se dá por treinamento em serviço, caracterizado pelo desenvolvimento de atividades assistenciais, sob supervisão, durante o internato e a residência médica. Em particular, os anos dedicados à residência médica se caracterizam por um período da formação médica muito produtivo, quando são adquiridas, desenvolvidas e consolidadas as habilidades e competências fundamentais para a prática médica. Sabe-se, no entanto, que a produção de conhecimento na área médica se dá em escala exponencial, o que exige constante atualização por parte do profissional de saúde. Portanto, a familiarização do médico com princípios de método científico é fundamental para garantir sua autonomia no gerenciamento da sua prática profissional. Porém, essa competência geralmente não é contemplada pelas matrizes curriculares dos programas de residência médica, tendo-se em vista seu caráter de treinamento em serviço.

Neste contexto, é esperado que, dentro da multiplicidade de processos necessários para a formação de médicos residentes, sejam oferecidas também condições, cenários, recursos e estratégias de aprendizagem que permitam o desenvolvimento de habilidades e competências complementares que propiciem autonomia na obtenção, interpretação, produção e aplicação do conhecimento científico e de novas tecnologias.
Programas de mestrado profissional integrados a programas de residência médica podem ser uma alternativa que venha a preencher essa lacuna na formação médica, garantindo a oferta aos serviços públicos e privados de saúde de profissionais efetivamente qualificados para, com base em evidências científicas, modificar positivamente as práticas em saúde e garantir uma assistência à saúde de excelência.

\section{CONCEITOS}

Mestrado profissional é uma modalidade de programa de pós-graduação stricto sensu, regulamentada por normativas governamentais (Portaria MEC n. ${ }^{\circ}$ 389, de 23 de março de 2017, Portaria CAPES n. ${ }^{\circ} 131$, de 28 de junho de 2017). Diferencia-se de programas acadêmicos pelo seu foco prioritário na capacitação de profissionais que atendam a demandas oriundas da prática profissional, nas diversas áreas do conhecimento. Neste contexto, a estrutura curricular de cursos de mestrado profissional deve contemplar a articulação entre conhecimento atualizado, metodologia pertinente e aplicação em campo de atuação profissional específico, com projeto e trabalho de conclusão do curso voltados para problemas reais da atuação profissional. Espera-se que o egresso de um curso/programa profissional adquira domínio do método científico que lhe permita buscar soluções para problemas identificados na prática profissional, através da geração de processos, procedimentos e produtos que contribuam para o avanço técnico, científico e tecnológico da sociedade, atuando como agente transformador de seu ambiente (www.capes.gov.br).

Mestrados profissionais na área médica diferenciam-se também de programas de residência médica, uma vez que estes constituem modalidade do ensino de pós-graduação lato sensu, sob a forma de curso de especialização, caracterizada por treinamento em serviço oferecido por instituições de saúde, universitárias ou não, sob a orientação de profissionais médicos de elevada qualificação ética e profissional (decreto $n .^{\circ}$ 80.281, de 05 de setembro de 1977). 


\section{CARACTERIZAÇÃO INSTITUCIONAL}

O Hospital das Clínicas da Faculdade de Ribeirão Preto da Universidade de São Paulo (HCFMRP-USP) é uma autarquia pública estadual, vinculada administrativamente à Secretaria Estadual da Saúde e associado à Faculdade de Medicina de Ribeirão Preto da Universidade de São Paulo para fins de ensino, pesquisa e assistência. Está inserido no Sistema Único de Saúde (SUS) como referência terciária/quaternária para uma população de cerca de quatro milhões de habitantes do nordeste do estado de São Paulo e outras regiões do Brasil. Oferece atendimento nas mais diversas especialidades médicas, possuindo recursos humanos, infraestrutura, laboratórios e equipamentos de saúde diversificados e de alta qualidade.

O Complexo de saúde HCFMRP-USP/FMRP-USP/FAEPA é composto por várias unidades próprias e conveniadas, distribuídas nos diferentes níveis de atenção à Saúde: Unidade Campus (742 leitos), Unidade de Emergência (176 leitos), Hospital Estadual de Ribeirão Preto (50 leitos), Centro de Referência da Saúde da Mulher (MATER, 45 leitos), Hospital Estadual de Américo Brasiliense (97 leitos), Hospital Estadual de Serrana (84 leitos), Centro de Saúde Escola Joel Domingos Machado, Centro Médico Social e Comunitário de Vila Lobato, Centro de Saúde Escola Ipiranga, Núcleos de Saúde da Família e Centro Médico Social e Comunitário Pedreira de Freitas, em Cássia dos Coqueiros. Em 2019, foram realizadas 583.367 consultas ambulatoriais e 107.145 procedimentos apenas na Unidade Campus (https://site.hcrp. usp.br/relatorios-de-atividades/), números esses que ilustram a efetiva inserção do Complexo HCFMRP-USP no Sistema Único de Saúde (SUS), nos diferentes níveis de atenção à Saúde. Neste contexto, os profissionais vinculados ao HCFMRP-USP estão cotidianamente em contato com as peculiaridades e necessidades locais do sistema público de saúde, com real possibilidade de proposição de intervenções, aplicação de novas tecnologias em saúde e avaliações de desfechos.

Conforme previsto desde a sua instituição como entidade autárquica (Lei n. ${ }^{\circ} 3.274$ de 23 de dezembro de 1955), o HCFMRP-USP é um hospital universitário e tem como uma das suas finalidades a) contribuir para a pesquisa e inves- tigação cientifica, sob todas as suas formas; b) servir de campo para a instrução de estudantes de medicina e enfermagem; c) servir de campo para aperfeiçoamento de médicos, na forma prevista no seu regulamento (https://site.hcrp.usp. br/leis-regimentos/).

O HCFMRP-USP tem longa tradição como campo de estágio para médicos residentes. Atualmente, conta com 1.055 vagas de residência médica credenciadas junto à Comissão Nacional de Residência Médica, distribuídas em 74 programas, sendo 21 programas de acesso direto, 24 de especialidades e 29 de áreas de atuação e 861 bolsas financiadas pela Secretaria Estatual de Saúde $(n=600)$ e pelo Ministério da Saúde $(n=$ 261). No último processo seletivo (2020), foram ofertadas 390 vagas/bolsas, incluindo programas de acesso direto $(n=212)$ e especialidades/áreas de atuação ( $n=178)$, que foram disputadas entre 2.633 candidatos aos programas de acesso direto e 1.148 aos programas de especialidade/ área de atuação. Trata-se de serviço de referência nacional, recebendo médicos residentes de todos os estados do país.

Esta integração entre inserção efetiva na rede pública de atenção à saúde e atuação ativa em produção técnico-científica ofereceu as condições institucionais ideais para a ampliação e diversificação do papel do HCFMRP-USP na formação de recursos humanos, através da proposição de programa de mestrado profissional vinculado aos programas de residência médica.

\section{PROPOSIÇÃO E IMPLANTAÇÃO DO PROGRAMA DE PÓS-GRADÚAÇÃO "MESTRADO PROFISSIONAL EM ME- DICINA" (MP MEDICINA)}

A atuação intensa do corpo docente do HCFMRP-USP na formação de médicos residentes apontou para a necessidade de ampliação das suas atividades acadêmicas, tendo-se em vista as demandas da sociedade para a oferta ao mercado de trabalho de médicos mais bem qualificados para a prática profissional. Conforme comentado anteriormente, o avanço exponencial no conhecimento científico exige profissionais com autonomia para a identificação de necessidades da prá- 
tica cotidiana e proposição de soluções realmente efetivas. Esta proposta de mestrado profissional associado a programas de residência médica foi elaborada no intuito de promover o desenvolvimento, por médicos residentes, de habilidades e competências que permitam a análise crítica dos processos de saúde e intervenções transformadoras da prática profissional.

As matrizes curriculares dos diferentes programas de residência médica do HCFMRP-USP contemplam atividades em cenários de saúde diversificados, em todos os níveis de atenção à saúde. Neste contexto, os supervisores e médicos residentes vinculados ao HCFMRP-USP estão cotidianamente em contato com as peculiaridades e necessidades locais do sistema público de saúde, com real possibilidade de proposição de intervenções e aplicação de novas tecnologias. Uma peculiaridade local, que diferencia a qualidade dos processos de ensino-aprendizagem implementados nos programas de residência médica do HCFMRP-USP, é a participação ativa e intensa de docentes da FMRP-USP, que, na sua vasta maioria, trabalham em regime de dedicação integral ao ensino e à pesquisa (RDIDP). Além dos docentes da FMRP-USP, o corpo docente dos programas de residência médica conta também com a participação de médicos assistentes do HCFMRP-USP, sendo uma parcela significativa detentores do título de doutor.

Informações detalhadas sobre o MP-Medicina estão disponibilizadas na página do programa (https://sites.usp.br/mpmed/). A seguir, serão discutidas as características mais relevantes do programa.

\section{PÚBLICO-ALVO}

O MP-Medicina destina-se, fundamentalmente, a médicos com vínculo atual, ou em passado recente, com os Programas de Residência Médica e Estágios de Complementação Especializada do HCFMRP-USP.

Considerando a diversidade de especialidades médicas envolvidas na proposta, o período ideal de entrada no programa de mestrado profissional é definido por cada programa de residência médica, de maneira a garantir que a formação específica da residência médica não seja comprometida. Há, no entanto, a sugestão de que a admissão ao mestrado profissional ocorra nos anos finais da residência, de maneira a não competir com as atividades programáticas da residência médica.

São previstos até dois processos seletivos por ano, com previsão de admissão de cerca de 60 alunos por ano. A estimativa de duração do programa é de 24 meses.

\section{CORPO DOCENTE}

O corpo docente é composto por docentes com sólida formação técnico-científica, associada à prática assistencial em saúde, com experiência na organização de serviços, estabelecimento de protocolos clínicos e desenvolvimento de novas tecnologias em saúde, e que sejam referências nas suas respectivas especialidades médicas. Vale reforçar que os orientadores são docentes de áreas clínicas e com efetiva atuação na formação de médicos residentes. O MP-Medicina conta, atualmente, com 30 orientadores plenos e três orientadores específicos.

Uma peculiaridade do MP-Medicina é seu caráter multidisciplinar, com a inclusão de orientadores e alunos com perfis variados, provenientes de várias áreas da medicina. O corpo docente atual é composto por docentes das áreas de Cirurgia, Clínica Médica, Fisiatria, Ginecologia e Obstetrícia, Medicina Social, Oftalmologia, Ortopedia, Otorrinolaringologia, Pediatria e Psiquiatria.

Espera-se que a multidisciplinaridade permita uma maior integração entre médicos (orientadores e alunos) provenientes de diferentes especialidades. O contato com diferentes saberes favorece a ampliação do leque de conhecimentos, habilidades e competências dos alunos, de maneira a garantir a oferta, ao mercado de trabalho, de profissionais versáteis e criativos na identificação e resolução de problemas.

\section{DISCIPLINAS}

É oferecido um conjunto vasto de disciplinas, atualmente no total de 21 , de maneira a 
atender à diversidade de necessidades e interesses de alunos provenientes de diferentes especialidades médicas. É preservado, no entanto, o caráter metodológico das disciplinas. As disciplinas são oferecidas em horários que não interfiram nas atividades regulares dos programas de residência médica. Também é estimulado o uso de estratégias de ensino à distância, desde que isso não interfira com as normas vigentes da instituição. Os orientadores do programa são estimulados para a proposição de disciplinas de caráter multidisciplinar que propiciem a efetiva integração entre diferentes saberes médicos.

Os alunos devem cumprir um mínimo de 20 créditos em disciplinas. Tendo em vista a heterogeneidade do público-alvo, foram estabelecidas como disciplinas obrigatórias (Bioética e Ética Médica e Sistemas de Informação em Saúde) apenas aquelas consideradas fundamentais para o desenvolvimento de habilidades e competências para a produção e aplicação de novas tecnologias em saúde e que sejam comuns às diferentes especialidades médicas envolvidas nessa proposta.

\section{LINHAS DE PESQUISA}

Em virtude da diversidade de especialidades médicas envolvidas na proposta, as linhas de pesquisa foram definidas de maneira a abranger os diferentes saberes e promover a articulação e integração entre médicos de diferentes especialidades, visando garantir um caráter efetivamente multidisciplinar para a proposta. Neste contexto, a presente proposta prevê quatro linhas de pesquisa, a saber:

Diagnóstico e Métodos de Investigação em Saúde (DMIS): Objetiva a proposição e avaliação da aplicabilidade, validade e efetividade de métodos diversificados de investigação clíni$\mathrm{ca}$, cirúrgica, laboratorial ou de imagem voltados para o aprimoramento do diagnóstico e estabelecimento de prognóstico de doenças. São incluídos nesta linha de pesquisa: desenvolvimento e aprimoramento de técnicas laboratoriais e kits para diagnóstico, proposição de instrumentos de avaliação e testes diagnósticos, utilização de procedimentos e técnicas invasivas, desenvolvimento de softwares e de algoritmos e avaliação de novas tecnologias em saúde.

Protocolos Clínicos e de Intervenções em Saúde (PCIS): Objetiva a elaboração, implantação e avaliação da efetividade de métodos diversificados de intervenção farmacológica, clínica, cirúrgica, psicossocial, comunitária e de reabilitação, cujo objetivo seja a remissão completa de doenças, controle sintomatológico, melhora da qualidade de vida e da funcionalidade global e das condições de saúde. Exemplos de estudos incluídos nesta linha de pesquisa são a verificação da efetividade e eficiência de fármacos, a análise de técnicas cirúrgicas, elaboração de protocolos clínicos para diagnóstico e tratamento de doenças, elaboração de protocolos de rastreamento e promoção de saúde, estadiamento, estabelecimento de prognóstico e resposta terapêutica.

Gestão em Saúde, Políticas Públicas e Avaliação de Serviços (GSPPAS): Objetiva a análise crítica das práticas assistenciais e de gerenciamento de serviços de saúde, proposição de novas formas de organização de serviços, verificação da qualidade da assistência prestada e investigações epidemiológicas. São incluídos nesta linha de pesquisa a verificação da resolutividade dos serviços, elaboração de protocolos regulatórios, estudo de estrutura, processos e resultados, estabelecimento de indicadores de saúde, avaliação de tratamentos com medicações especiais de alto custo e estudos de farmacovigilância e farmacoeconomia.

\section{Ensino e Aprendizagem em Saúde} (EAP): Objetiva o estudo dos diversos processos de aprendizado em Saúde e seus resultados. São incluídos nesta linha de pesquisa estudos relativos à implantação, caracterização e avaliação da efetividade de estratégias inovadoras de ensino e aprendizagem, avaliação formativa e somativa dos alunos, estimativa de resultados, definição do perfil do egresso, inserção no mercado de trabaIho e detecção de demandas locais.

\section{OBJETIVOS TERMINAIS DO PROGRAMA}

Espera-se que, ao final do Programa de Pós-Graduação "Mestrado Profissional em Medi- 
cina", o médico desenvolva habilidades e competências que o capacitem a modificar e aprimorar as práticas em saúde, por meio de:

a) Análise crítica de práticas assistenciais em saúde, com base em dados científicos atuais;

b) Detecção de necessidades locais e proposição de soluções factíveis, considerando os recursos disponíveis;

c) Proposição e implantação de estratégias inovadoras de planejamento, criação e uso de novas tecnologias e sistematização de protocolos de intervenção em saúde;

d) Autonomia para o aprimoramento e resolução de problemas de práticas de saúde;

e) Participação no delineamento de políticas públicas em saúde.

\section{PRODUTO FINAL}

A conclusão do curso se dá por depósito de material escrito e defesa pública de dissertação de mestrado, relacionada ao desenvolvimento de um produto final de diferentes modalidades, a saber: a) produção bibliográfica (artigo publicado em revista técnica ou revista de divulgação); b) processo ou produto com potencial patenteável; c) cursos de capacitação profissional; d) editoração (organização de livros, coletâneas, revistas, anais); e) material didático; f) softwares/ aplicativos; g) normas ou marcos regulatórios; h) relatórios técnicos conclusivos; i) manuais ou protocolos de atenção à saúde; j) programas de divulgação na mídia.

\section{IMPLANTAÇÃO DO PROGRAMA}

A proposta do MP-Medicina foi aprovada pela Pró-Reitoria de Pós-Graduação da USP em setembro de 2017 e pela Coordenação de Aperfeiçoamento de Pessoal de Nível Superior (CAPES) em outubro de 2018. A partir disso, foi constituída a Comissão Coordenadora do Programa (CCP), composta por quatro membros titulares e respectivos suplentes, eleitos pelos orientadores cre- denciados no programa. Conforme as normas institucionais, a representação discente, eleita pelos pares, também é membro componente da CCP. O programa iniciou formalmente suas atividades em maio de 2019, com a admissão da primeira turma de alunos.

A admissão de novos alunos se dá por concurso público. O processo seletivo baseia-se na avaliação escrita e arguição oral de projeto de pesquisa, elaborado sob supervisão de um dos orientadores credenciados no programa, e arguição de currículo. Os critérios de avaliação são previstos em editais aprovados pela instituição-sede do programa. Até o momento, foram realizados quatro processos seletivos.

Desde o início das atividades do programa, foram admitidos sessenta alunos, sendo a primeira defesa de mestrado de aluno do programa realizada em maio de 2020. Os objetos de estudo dos projetos de pesquisa desenvolvidos pelos alunos são variados, com a perspectiva de desenvolvimento de processos e produtos com potencial de transformação da prática profissional em assistência em saúde. Como exemplos, podem ser citados desenvolvimento, revisão e atualização de protocolos de diagnóstico e tratamento clínico e cirúrgico, caracterização de população usuária e de organização de serviços, e desenvolvimento de material didático para capacitação de profissionais de saúde.

Do ponto de vista organizacional, a Comissão Coordenadora do Programa procura manter contatos regulares com alunos e orientadores e promover atividades coletivas, de maneira a garantir a integração entre os diferentes saberes e a consolidação do programa, em esforços contínuos e coletivos para atingir níveis de excelência na formação do corpo discente e oferta de produtos que, de fato, tragam melhorias para a sociedade.

\section{CONSIDERAÇÕES FINAIS}

Conciliar as necessidades e peculiaridades das diferentes especialidades médicas que compõem o programa e, ao mesmo tempo, garantir a efetiva articulação entre os diferentes saberes é um grande desafio. Por outro lado, a diversidade tem se mostrado um campo profícuo para a facili- 
tação do desenvolvimento das habilidades e competências esperadas do egresso do programa.

Ademais, combinar a formação prática, em treinamento em serviço, como é característico de programas de residência médica, com a formação em metodologia científica é uma tarefa comple$\mathrm{xa}$, que exige novos aprendizados dos próprios orientadores do programa. Este esforço torna-se inevitável, uma vez que produção técnica/tecnológica de relevância efetiva para a assistência e políticas públicas de saúde é indissociável da produção científica qualificada.

Um desfecho potencialmente positivo de programa de mestrado profissional vinculado a programas de residência médica é a possibilidade de incentivo a médicos efetivamente vocacionados para atividades acadêmicas para o aprofunda- mento de sua formação científica e consequente engajamento em programas de doutorado acadêmico da unidade. Esse estímulo ganha ainda mais relevância tendo-se em vista o longo tempo de formação da carreira médica, uma vez que a formação stricto sensu inicial (mestrado) pode ser feita em paralelo à conclusão da formação lato sensu (residência médica).

Concluindo, a experiência inicial do programa vem confirmando a possibilidade de ampliação do leque de habilidades a serem desenvolvidas durante a formação médica, com a perspectiva de ofertar ao mercado de trabalho profissionais dotados de autonomia e capacidade de reflexão crítica, que possam efetivamente modificar e aprimorar a prática profissional e a assistência aos pacientes.

Autor Correspondente: Cristina Marta Del-Ben delben@fmrp.usp.br

Editor:

Prof. Dr. Marcelo Riberto

Recebido: $14 / 10 / 2020$

Aprovado: 13/07/2021

(c) (i) Este é um artigo publicado em acesso aberto (Open Access) sob a licença Creative

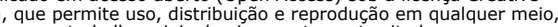
sem restrições, desde que o trabalho original seja corretamente citado. 